\title{
Adding complex terrain and stable atmospheric condition capability to the OpenFOAM-based flow solver of the simulator for on/offshore wind farm applications (SOWFA)
}

\author{
Matthew J. Churchfield ${ }^{a}$, Sang Lee, and Patrick J. Moriarty \\ National Renewable Energy Laboratory, 15013 Denver West Parkway Golden, CO 80401, USA
}

\begin{abstract}
The National Renewable Energy Laboratory's Simulator for On/Offshore Wind Farm Applications contains an OpenFOAM-based flow solver for performing large-eddy simulation of flow through wind plants. The solver computes the atmospheric boundary layer flow and models turbines with actuator lines. Until recently, the solver was limited to flows over flat terrain and could only use the standard Smagorinsky subgrid-scale model. In this work, we present our improvements to the flow solver that enable us to 1) use any OpenFOAM-standard subgrid-scale model and 2) simulate flow over complex terrain. We used the flow solver to compute a stably stratified atmospheric boundary layer using both the standard and the Lagrangian-averaged scale-independent dynamic Smagorinsky models. Surprisingly, the results using the standard Smagorinsky model compare well to other researchers' results of the same case, although it is often said that the standard Smagorinsky model is too dissipative for accurate stable stratification calculations. The scale-independent dynamic subgrid-scale model produced poor results, probably due to the spikes in model constant with values as high as 4.6. We applied a simple bounding of the model constant to remove these spikes, which caused the model to produce results much more in line with other researchers' results. We also computed flow over a simple hilly terrain and performed some basic qualitative analysis to verify the proper operation of the terrain-local surface stress model we employed.
\end{abstract}

\section{Introduction}

In this work, we present improvements to the OpenFOAM-based Simulator for On/Offshore Wind Farm Applications (SOWFA), that is being continually developed at the U.S. Department of Energy's National Renewable Energy Laboratory (NREL). SOWFA is composed of an OpenFOAM-based incompressible atmospheric/wind farm large-eddy simulation (LES) solver that models turbines as actuator lines coupled with NREL's FAST wind turbine structural and system dynamics model. An example of a flow computed with SOWFA is that of the 48-turbine Lillgrund offshore wind farm that lies between Sweden and Denmark [1].

Until recently, SOWFA was limited to computing wind farm flow over flat terrain under neutral or unstable atmospheric conditions. The flat terrain limitation existed because we had implemented planetary surface shear stress and temperature flux models commonly used in the atmospheric LES

\footnotetext{
a e-mail: matt . churchfield@nrel.gov
}

This is an Open Access article distributed under the terms of the Creative Commons Attribution License 2.0, which permits unrestricted use, distribution, and reproduction in any medium, provided the original work is properly cited. 
community that rely on Monin-Obukhov similarity theory. The surface stress models assume flat homogeneous terrain over which a planar average wind profile is calculated that is then used to determine the average planetary surface stress. The limitation that only neutral and unstable atmospheric conditions could be simulated was due to the fact that the flow solver relied on a cell face-based subgrid-scale (SGS) viscosity formulation, which deviates from the OpenFOAM-standard cell-centered turbulence variable approach. This means that the OpenFOAM-standard SGS models were not compatible with our custom LES solver. Because of its simplicity, we implemented only the standard Smagorinsky model into this nonstandard face-based SGS formulation, but Brown et al. [2] showed that the standard Smagorinsky model does not perform as well as models with backscatter in simulating stable atmospheric flow. Furthermore, Beare et al. [3] have shown that at typical grid resolution, SGS models more sophisticated than the standard Smagorinsky model are able to better capture stable atmospheric boundary layer details. Also, the Lagrangian-averaged scale-dependent dynamic Smagorinsky model of Stoll and PortéAgel [4] has been shown to perform well in stably stratified atmospheric flows [5].

To circumvent these limitations, we implemented a local planetary surface stress model that does not require horizontal averages in a plane of homogeneity, following the work of Wan and colleagues [6]. This allows the solver to compute flow over irregular terrain. To create a terrain-conforming mesh, we use OpenFOAM's moveDynamicMesh solver. We also migrated back to the standard OpenFOAM cell-centered SGS viscosity approach, and the results are very similar to our previous face-centered SGS stress approach, meaning that the entire suite of standard OpenFOAM SGS models can now be used with our atmospheric/wind farm flow solver, including the Lagrangian-averaged scale-independent dynamic Smagorinsky model of Meneveau and colleagues [7].

With these enhancements to the SOWFA flow solver, we computed the well-documented stably stratified atmospheric boundary layer computed in the Global Energy and Water Cycle Experiment (GEWEX) Atmospheric Boundary Layer Study (GABLS) model intercomparison [3]. We also computed the flow of a neutral boundary layer capped with an inversion over a simple hilly terrain.

We are interested in simulating stably stratified flow because it can be one of the most damaging flows for wind turbines. Kelley [8] spent many years at NREL researching the effects of the stable atmospheric boundary layer (ABL) on wind turbines. His study shows that much wind turbine damage occurs in weakly stable flow. Stable ABL flows are characterized by lower and sometimes intermittent turbulence levels and strong vertical shear of wind speed and direction, both of which cause considerable fatigue loads on modern turbine rotors with a span on the order of $100 \mathrm{~m}$. The lower turbulence levels mean that turbine wakes persist for longer distances downstream, and hence can decrease the efficiency of a wind plant. The stable ABL can also contain a low-level jet, a layer of flow that has speed greater than the flow above the ABL. Gravity waves can also form. All of these unique features of the stably stratified ABL make it important and challenging to simulate accurately.

Flow through complex terrain is important because effects like local acceleration, separation, and recirculation can occur, all of which have important impacts on turbines located within the terrain. Although many wind plants are located in flat regions, such as those in the U. S. Midwest region, there is a significant number of farms located in complex terrain. Complex terrain remains a challenge for reduced-order flow models used by wind plant layout engineers, so having an accurate computational tool would help to improve these reduced-order tools.

\subsection{History of subgrid-scale models suitable for the stable atmospheric boundary layer and terrain}

Subgrid-scale models are an important part of LES of the ABL. Near the lower surface, where the turbulent scales are constrained and the largest resolved eddies become smaller than at greater distances from the surface, the influence of the SGS model is more dominant than at greater distances from the surface. The same is true of LES of stable ABLs. Because buoyancy forces act to suppress turbulence 
in those cases, the largest resolved scales are smaller than those resolved in LES of neutral or unstable ABLs. SGS models often assume that the subgrid scales of turbulence are fairly isotropic, which is not true of the near-wall or stable ABL subgrid scales for typical grid resolutions. Dynamic SGS models have been seen as an improvement over static models, but they rely on some type of averaging, which often is done in the direction of homogeneity. In flow over complex terrain, there is no direction of homogeneity, so again, a well-designed SGS model is important. Because of their important role in LES of the ABL, we will discuss the lineage of SGS models that have led to some of today's most sophisticated models that are used for LES of the stable ABL and of the ABL over terrain.

In performing LES of the ABL, often the incompressible filtered Navier-Stokes equations are solved, using the Boussinesq approximation for buoyancy, along with the continuity equation, which is usually enforced through the solution of an elliptic equation for the pressure variable. The continuity equation is

$$
\frac{\partial \bar{U}_{i}}{\partial x_{i}}=0
$$

and the momentum equation is

$$
\frac{\partial \bar{U}_{i}}{\partial t}+\frac{\partial \bar{U}_{j} \bar{U}_{i}}{\partial x_{j}}=-2 \epsilon_{i 3 k} \Omega \bar{U}_{k}-\frac{\partial \bar{p}}{\partial x_{i}}-\frac{\tau_{i j}}{\partial x_{j}}+\rho_{b} g_{i} .
$$

In these equations, the over line denotes the LES filtering operation, $\bar{U}_{i}$ is the component of the resolvedscale velocity vector in the coordinate direction $x_{i}, \epsilon_{i j k}$ is the alternating unit tensor, $\Omega$ is the planetary rotation rate vector at the point of interest on the planet (which is dependent on latitude), $\bar{p}$ is pressure, $\tau_{i j}$ is the SGS stress tensor, $\rho_{b}$ is a scalar that dictates the sign and strength of the buoyancy force, and $g_{i}$ is the gravitation vector. In order to compute $\rho_{b}$, which is given by

$$
\rho_{b}=1-\left(\frac{\bar{\theta}-\theta_{0}}{\theta_{0}}\right),
$$

where $\bar{\theta}$ is the resolved-scale potential temperature and $\theta_{0}$ is a reference temperature, a potential temperature transport equation must be solved and is given by

$$
\frac{\partial \bar{\theta}}{\partial t}+\frac{\partial \bar{U}_{j} \bar{\theta}}{\partial x_{j}}=-\frac{\tau_{\theta i}}{\partial x_{i}},
$$

where $\tau_{\theta i}$ is the SGS temperature flux. In both the momentum and potential temperature equations, the effects of molecular diffusion are not included because the SGS effects are much more dominant, except very near the surface. Near the surface, LES of the ABL nearly always relies on some sort of surface model in which viscous and SGS stresses and temperature fluxes are lumped together.

The SGS stress tensor arises from the filtering of the Navier-Stokes equations. The first term of the right-hand side of $\tau_{i j}=\overline{U_{i} U_{j}}-\bar{U}_{i} \bar{U}_{j}$ cannot be directly solved, so $\tau_{i j}$ must be modeled. The majority of SGS models rely upon the linear eddy-viscosity assumption that the SGS stress found in Eq. (2) is related to resolved-scale strain by

$$
\tau_{i j}^{D}=-2 v_{t} \bar{S}_{i j}
$$

where $\tau_{i j}^{D}$ is the deviatoric SGS stress tensor (in practice, the isotropic part can be absorbed into the pressure gradient term of the momentum equation), $v_{t}$ is the SGS viscosity, and

$$
\bar{S}_{i j}=\frac{1}{2}\left(\frac{\partial \bar{U}_{i}}{\partial x_{j}}+\frac{\partial \bar{U}_{j}}{\partial x_{i}}\right)
$$


is the resolved-scale strain rate tensor. The SGS temperature flux vector in Eq. (4) must be modeled and is often done in a similar manner to the SGS stress tensor using

$$
\tau_{\theta i}=-\frac{\nu_{t}}{\operatorname{Pr}_{t}} \frac{\partial \bar{\theta}}{\partial x_{i}},
$$

where $\operatorname{Pr}_{t}$ is the turbulent Prandtl number.

In the relations of Eqs. (5) and (7), $v_{t}$ and $\operatorname{Pr}_{t}$ must be prescribed. Smagorinsky [9] devised one of the earliest models for $v_{t}$,

$$
v_{t}=\left(C_{s} \bar{\Delta}\right)^{2} \bar{S},
$$

in which $C_{s}$ is a fixed constant and $\bar{\Delta}$ is usually related to the mesh as $\bar{\Delta}=(\Delta x \Delta y \Delta z)^{1 / 3}$, where $\Delta x$, $\Delta y$, and $\Delta z$ are the mesh cell lengths in the $x$-, $y$-, and $z$-directions, respectively, and $\bar{S}=\left(2 \bar{S}_{i j} \bar{S}_{i j}\right)^{1 / 2}$. This model was derived using the assumption that the shear-driven production and dissipation of SGS kinetic energy are in balance, which does not always occur. For example, the equilibrium does not occur in the unstable buoyancy-driven ABL. Another important disadvantage of the standard Smagorinsky model is that a given value of $C_{s}$ is not necessarily ideal in all locations of the flow. For example, near the planetary surface where shear dominates the turbulence production, the ideal value of $C_{s}$ is different than higher into the boundary layer. For different applications, researchers have given values of $C_{s}$ ranging from about 0.06 to 0.2 , with the smaller values used in shear-driven flow.

An alternative to the standard Smagorinsky model, which allows for an imbalance of shear-driven production and dissipation of SGS kinetic energy, is to base the SGS model on a partial differential equation for SGS kinetic energy, such as the model used by Moeng [10]. Once SGS kinetic energy is computed, then $v_{t}$ can be found using

$$
v_{t}=C_{k} l e^{1 / 2},
$$

where $C_{k}$ is a model constant, $e$ is the SGS kinetic energy, and $l$ is a length scale given by

$$
l=\frac{0.76 e^{1 / 2}}{\left(\frac{g}{\theta_{0}} \frac{\partial \bar{\theta}}{\partial z}\right)^{1 / 2}},
$$

where $g$ is magnitude of the gravitation vector.

There have been attempts to modify the standard Smagorinsky model. One of the more notable modifications is given by Mason and Derbyshire [11] in which the model includes a function in which the filter length scale is reduced near the surface. Another function modifies both the length and SGS velocity scale based on the local flux Richardson number. They used this model with limited success in their pioneering LES of the stable ABL. Because the Smagorinsky model is purely dissipative, Mason and Thompson [12] further modified the Mason and Derbyshire version of the Smagorinsky model by including the effect of "backscatter" of turbulence energy from small scales to large scales. This is done by introducing random accelerations and fluxes into the momentum and temperature transport equations that have magnitudes that give the desired backscatter rates. They show that backscatter improves the predictions of mean velocity shear near the surface, which is an important and common problem in LES of the ABL

There have also been attempts to modify the SGS kinetic energy based model, an important example being the work of Sullivan, McWilliams, and Moeng [13]. They start with the SGS kinetic energy model used by Moeng [10] and replace the stress-strain relationship given in Eq. (5) with

$$
\tau_{i j}^{D}=-2 v_{t} \gamma \bar{S}_{i j}-2 v_{T}\left\langle\bar{S}_{i j}\right\rangle
$$

In this equation, $\gamma$ is an "isotropy factor" that accounts for the variability in SGS constants due to anisotropy of the mean flow, and it controls the transition between SGS and ensemble-averaged 
turbulence parameterizations. Such a transition occurs near the surface where the energy-containing turbulent scales become small enough that the mesh does not well capture them and the resolved flow approaches ensemble-averaged flow. The angle-brackets denote averaging over the homogeneous directions, which are the horizontal directions in ABL flows over flat terrain. Sullivan et al. [13] show that this modified one-equation SGS model improves the predictions of mean velocity shear near the surface as compared to the baseline one-equation model. Andren [14] showed that the model of Sullivan et al. is about as successful as the Smagorinsky model with backscatter of Mason and Thompson [12], and he used the model to simulate the stably-stratified ABL.

Kosović [15] developed a nonlinear relationship to replace Eq. (5) for use with the one-equation SGS kinetic energy model that accounts for anisotropy due to both shear and backscatter. Kosović and Curry [16] later used the nonlinear backscatter model to simulate the stably stratified ABL.

A different approach than the ones listed above to modify the standard Smagorinsky model is to make the model constant dynamic such that it varies in space and time based on the flow. Germano et al. [17] introduced this approach. The SGS stresses at a second "test" filter scale (typically two times as large as the base filter width usually associated with the mesh cell volume), denoted by an over tilde, are

$$
T_{i j}=\widetilde{U_{i} U_{j}}-\tilde{\bar{U}}_{i} \tilde{\bar{U}}_{j}
$$

The Germano identity is that

$$
L_{i j}=T_{i j}-\tilde{\tau}_{i j}=\widetilde{\bar{U}_{i} \bar{U}_{j}}-\tilde{\tilde{U}_{i}} \tilde{\bar{U}}_{j} .
$$

All quantities that form the tensor $L_{i j}$ can be found so $L_{i j}$ can be directly computed. If $T_{i j}$ and $\tilde{\tau}_{i j}$ are modeled using the standard Smagorinsky model, then they are

$$
\begin{gathered}
T_{i j}=-2\left(C_{s}(\tilde{\Delta}) \tilde{\Delta}\right)^{2} \tilde{\bar{S}}_{\overline{\bar{S}}}, \\
\tilde{\tau}_{i j}=-2\left(C_{s}(\bar{\Delta}) \bar{\Delta}\right)^{2} \widetilde{\bar{S}} \widetilde{\bar{S}}_{i j} .
\end{gathered}
$$

The error caused by modeling $L_{i j}$ with the Smagorinsky model can be expressed as

$$
e_{i j}=L_{i j}-2\left[\left(C_{s}(\bar{\Delta}) \bar{\Delta}\right)^{2} \widetilde{S}_{i j}-\left(C_{s}(\tilde{\Delta}) \tilde{\Delta}\right)^{2} \tilde{\bar{S}}_{\bar{S}}\right]
$$

and must be minimized. By assuming that $C_{s}$ is scale invariant $\left(C_{S}(\bar{\Delta})=C_{s}(\tilde{\Delta})\right)$, Lilly showed that the mean square error can be minimized by solving

$$
C_{s}^{2}=\frac{M_{i j} L_{i j}}{M_{k l} M_{k l}}
$$

where $M_{i j}$ is

$$
M_{i j}=2\left[\bar{\Delta}^{2} \widetilde{\bar{S}}_{i j}-\tilde{\Delta}^{2} \tilde{\bar{S}}_{\overline{\bar{S}}} \tilde{i}_{i j}\right] .
$$

In this way, $C_{s}$ is found at every point and time in the flow. However, this local scale-invariant dynamic method is numerically unstable, so in practice, Eq. (17) is solved as

$$
C_{s}^{2}=\frac{\left\langle M_{i j} L_{i j}\right\rangle}{\left\langle M_{k l} M_{k l}\right\rangle},
$$

where the angle-brackets denote some type of averaging. Often, the averaging is done over planes of homogeneity, as with the planar-averaged scale-independent (PASI) dynamic model, which is fine for flow over flat terrain. If the terrain is complex, though, such an averaging procedure does not make sense.

Meneveau et al. [7] proposed a Lagrangian-averaged scale-independent (LASI) dynamic Smagorinsky model in which the angle-brackets in Eq. (19) denote averaging backward along a 
streamline. They chose an exponential weighting for the averaging with strongest weighting at the point of interest. They denote the averaged quantities as $\mathscr{I}_{L M}=\left\langle M_{i j} L_{i j}\right\rangle$ and $\mathscr{I}_{M M}=\left\langle M_{k l} M_{k l}\right\rangle$, so then $C_{s}^{2}=\mathscr{I}_{L M} / \mathscr{I}_{M M}$. Because of the exponential weighting function, the averaging can be achieved by solving the equations

$$
\begin{gathered}
\frac{\partial \mathscr{I}_{L M}}{\partial t}+\frac{\bar{U}_{j} \mathscr{I}_{L M}}{\partial x_{j}}=\frac{1}{\theta \bar{\Delta}\left(\mathscr{I}_{L M} \mathscr{I}_{M M}\right)^{-1 / 8}}\left(L_{i j} M_{i j}-\mathscr{I}_{L M}\right), \\
\frac{\partial \mathscr{I}_{M M}}{\partial t}+\frac{\bar{U}_{j} \mathscr{I}_{M M}}{\partial x_{j}}=\frac{1}{\theta \bar{\Delta}\left(\mathscr{I}_{L M} \mathscr{I}_{M M}\right)^{-1 / 8}}\left(M_{i j} M_{i j}-\mathscr{I}_{M M}\right)
\end{gathered}
$$

where $\theta$ is a constant set to 1.5 .

The assumption of scale invariance of the model constant $C_{S}$ has been shown not to be fully valid and also results in under-dissipative SGS model behavior in LES of the ABL, so Porté-Agel et al. [18] devised a planar-averaged scaled-dependent (PASD) version of the dynamic Smagorinsky model. The model is more complicated than the scale-independent version because a fifth-order polynomial must be solved in each averaging plane, and the assembly of the polynomial coefficients requires computation of 10 distinct planar averaged quantities. The PASD model produces better neutral ABL results than the PASI model and is slightly more dissipative, which is desirable.

To remove the constraint of planar averaging but take advantage of the desirable features of the PASD model, Bou-Zeid et al. [19] devised a Lagrangian-averaged scale-dependent (LASD1) dynamic Smagorinsky model. If the same approach as the one taken by Porte-Agel et al. [18] in creating the PASD model were used, but the planar averages were replaced by Lagrangian averages, 10 Lagrangian averages would need to be computed at each point in the flow field during each time step, which would be expensive and possibly numerically unstable. Therefore, Bou-Zeid et al. [19] realized that when using the LASI or PASI model, the value of $C_{s}$ obtained is really more appropriate for the test filter scale $\tilde{\Delta}$ than the actual filter scale $\bar{\Delta}$. Knowing that fact, they were able to devise the LASD1 model such that only four Lagrangian averages are needed at each point during every time step. The model retains the good qualities of the PASD model but can be used over complex terrain.

Stoll and Porté-Agel [4] also devised a Lagrangian-averaged scale-dependent (LASD2) model that, unlike Bou-Zeid et al. [19], follows the original PASD model approach and requires the solution of 10 Lagrangian averages and a fifth-order polynomial at each point in the flow field during each time step. Further, they propose a method of dynamically solving for $C_{s}^{2} / \operatorname{Pr}_{t}$ for the SGS temperature flux, which requires the solution to a further 10 Lagrangian averages and a fifth-order polynomial at each point in the flow field during each time step.

Basu and Porté-Agel [20] present results of the LES of a stably stratified ABL using a locallyaveraged scale-dependent dynamic Smagorinsky model. The model works the same as the LASD2 model of Stoll and Porté-Agel [4], but the Lagrangian averages are replaced by local averages done in horizontal planes using a stencil of three by three grid points. They show that this procedure performs well in stably stratified ABL simulations and could be used over complex terrain. Later, Stoll and Porté-Agel [5] examine the effects of different averaging schemes used in scale-dependent dynamic Smagorinsky models when simulating the stably stratified ABL.

The specification of the turbulent Prandtl number, $\operatorname{Pr}_{t}$, that appears in Eq. (7) is also important. Some researchers simply specify a constant value of $\operatorname{Pr}_{t}$. Moeng [10] used the formula

$$
\left.\operatorname{Pr}_{t}=\left(1+\frac{2 l}{\bar{\Delta}}\right)\right)^{-1},
$$

where $l$ is defined in Eq. (10). In neutral and unstable flow $\operatorname{Pr}_{t}=1 / 3$, but as the flow becomes more stably stratified, $\operatorname{Pr}_{t}$ approaches 1. As mentioned above, Stoll and Porté-Agel [4] and Basu and PortéAgel [20] proposed not to specify $\operatorname{Pr}_{t}$, but rather to dynamically specify $C_{s}^{2} / \operatorname{Pr}_{t}$. 


\subsection{Past stable atmospheric boundary layer and terrain large-eddy simulation}

In this section, we briefly mention past work in simulating stably stratified ABL flows and ABL flows over terrain using LES. The pioneering stably stratified ABL LES was conducted by Mason and Derbyshire [11] in 1990. Other researchers who followed in performing LES of the stable ABL include Brown et al. [2], Andren [14], Kosović and Curry [16], Beare and MacVean [21], Basu and Porté-Agel [20], and Beare et al. [3]. Kleissl [22] performed a comparison of various dynamic Smagorinsky models in nonneutral ABL conditions. Stoll and Porté-Agel [5] performed stable ABL simulations with the scale-dependent dynamic Smagorinsky model using various SGS model averaging schemes.

Wan et al. [6], Sullivan et al. [23], and Kirkil et al. [24] are a few researchers who have worked on LES of ABL flow over terrain.

\section{Method}

The solver used is a customized version of OpenFOAM 2.1.x's buoyantBoussinesqPimpleFoam that includes modifications to include Coriolis forces, a large-scale driving-pressure gradient to achieve a desired wind speed at a given height, and specified surface stresses and temperature fluxes. The solver solves the momentum equation shown in Eq. (2), although in a slightly rearranged form because the pressure variable computed is the deviation from the hydrostatic pressure and is lumped with the isotropic part of the SGS stress tensor, an elliptic pressure equation to enforce continuity, and the potential temperature equation in Eq. (4). We used either the standard Smagorinsky model [9] or the LASI dynamic Smagorinsky model [7], both of which are included with OpenFOAM 2.1.x. Lapoint-Thériault [25] found that better temperature-velocity coupling is achieved by performing outer iterations, but the outer iterations are expensive. Therefore, we modified the solver to have not only a temperature predictor, but also correctors, in order to improve the coupling following Oliveira and Issa [26].

All cases in this study are periodic, so the lateral boundaries are cyclic. The upper boundary is set as a no-slip, zero stress boundary, the pressure gradient is specified depending on the Boussinesq density $\left(\rho_{b}\right)$ gradient normal to the boundary (OpenFOAM's "buoyantPressure" boundary condition), and the potential temperature gradient is set to match that of the initial temperature profile. At the lower boundary, the pressure gradient is set in the same way as at the upper boundary. Models for surface stress and temperature flux are used at the lower boundary, meaning that velocity parallel to the boundary and temperature need not be explicitly specified because their surface values do not enter the discretized momentum equation. Velocity normal to the boundary must be zero. The surface-normal gradient of velocity is needed, though, at the cell centers of the first layer of cells adjacent to this boundary in order to compute the strain-rate magnitude for the SGS model. Therefore, the surface velocity parallel to the wall is specified such that the cell center surface-normal gradient is equal to the gradient at the cell face opposite the boundary face (i.e. at the top of the cell). The standard Smagorinsky model does not require boundary conditions, but the LASI dynamic Smagorinsky model does for the model quantities $\mathscr{I}_{L M}$ and $\mathscr{I}_{M M}$. The boundary conditions for those two quantities are cyclic on the lateral boundaries and zero normal gradient at the upper and lower boundaries.

The surface shear stress model is that of Schumann [27]. At the surface, the stress tensor components are zero except for $\tau_{13}$ and $\tau_{23}$. They are specified as

$$
\begin{gathered}
\tau_{13}=-u_{*}^{2} \frac{\bar{U}_{1}\left(z_{1}\right)}{\left|\left\langle\bar{U}\left(z_{1}\right)\right\rangle\right|} \\
\tau_{23}=-u_{*}^{2} \frac{\bar{U}_{2}\left(z_{1}\right)}{\left|\left\langle\bar{U}\left(z_{1}\right)\right\rangle\right|},
\end{gathered}
$$




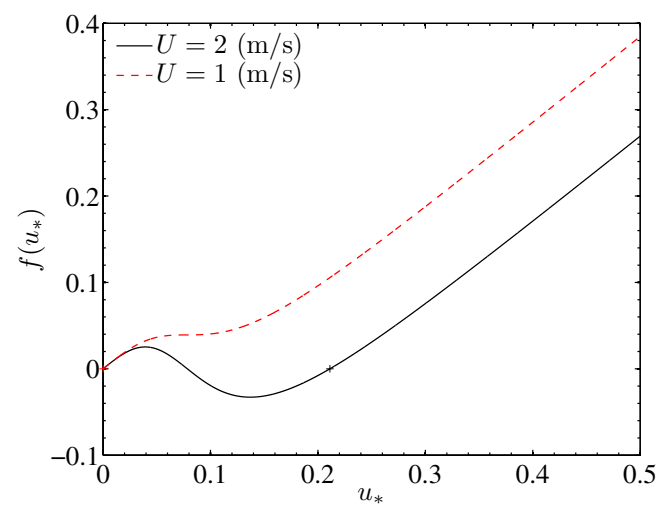

Figure 1. An illustration of the fact that when solving for $u_{*}$ for stably stratified flow, depending on the conditions, the only solution is that $u_{*}=0$, as in the case shown with the red dashed line.

where $u_{*}$ is the friction velocity, $z_{1}$ denotes the height of the center of the cells adjacent to the wall, and angle brackets denote a horizontal average. The friction velocity is found using Monin-Obukhov scaling. If the atmospheric stability is not neutral, the Monin-Obukhov scaling law becomes an implicit function of $u_{*}$. The root of the function must be found to determine $u_{*}$. The implicit function is

$$
f\left(u_{*}\right)=u_{*}-\frac{\kappa\left|\left\langle\bar{U}\left(z_{1}\right)\right\rangle\right|}{\log \left(\frac{z_{1}}{z_{0}}\right)-\psi_{M}\left(u_{*}\right)},
$$

where $\kappa$ is the von Kármán constant, $z_{0}$ is the aerodynamic roughness height, and $\psi_{M}$ is a stability parameter that is a function of various quantities including $u_{*}$. Normally, there are multiple roots to the function $f$ and the largest one is chosen. However, we found that for stable stratifications, at times the value of $\left|\left\langle\bar{U}\left(z_{1}\right)\right\rangle\right|$ would become low enough that the only root is $u_{*}=0$, which is not realistic. Nonetheless, we allowed the solver to at times have zero surface stress, meaning that $\left|\left\langle\bar{U}\left(z_{1}\right)\right\rangle\right|$ increased to the point that $u_{*}$, and hence surface stress, became finite again. This problem is illustrated in Figure 1 for two different values of $\left|\left\langle\bar{U}\left(z_{1}\right)\right\rangle\right|$, one of which has only the zero root, and the other has non-zero finite roots.

The surface temperature flux can be prescribed directly, or a surface cooling rate can be specified; we did the latter. Surface temperature flux is determined using the Monin-Obukhov scaling laws for velocity and potential temperature, which account for the difference between surface temperature and $\langle\bar{\theta}\rangle\left(z_{1}\right)$. At each time step, this surface temperature flux is specified uniformly over the lower surface. This method is discussed at length by Basu and colleagues [28].

A problem arises in complex terrain as the planar averages seen in Schumann's surface stress model or the Monin-Obukhov scaling laws no longer make sense. We follow many other researchers, such as Wan et al. [6], and apply Schumann's model and the scaling laws locally, although this may not be the best solution. Monin-Obukhov scaling laws were neither meant to be used locally, a point that Stoll and Porté-Agel [4] discuss, nor were they formulated for use over complex terrain. Another problem arises in that in complex terrain, the surface stress tensor must be computed in terrain local coordinates, specifying $\tau_{1^{\prime} 3^{\prime}}$ and $\tau_{2^{\prime} 3^{\prime}}$, where the primes denote the local coordinate system in which the $1^{\prime}$ direction is along the flow, $3^{\prime}$ is normal to the surface, and $2^{\prime}$ is orthogonal to $1^{\prime}$ and $3^{\prime}$. Once the local stress tensor is formed, it must then be transformed back into the Cartesian coordinates used by the flow solver. Dealing with terrain local coordinates and the surface stress tensor is discussed by Wan and colleagues [6]. 


\section{Results}

\subsection{Stable atmospheric boundary layer}

To test our method in simulating the stable ABL, we used the case of the GABLS model intercomparison $[3,29]$. The domain has a flat lower surface and is $400 \mathrm{~m} \times 400 \mathrm{~m} \times 400 \mathrm{~m}$. The domain is periodic in the lateral directions; boundary conditions, as described above, are applied at the lower surface; and a rigid, stress-free lid is used on the top boundary. The grid size is varied from $64^{3}$ to $128^{3}$. The geostrophic wind is specified as $U_{g}=(8.0,0.0,0.0) \mathrm{m} / \mathrm{s}$ and the simulation takes place at $73^{\circ}$ north latitude. A surface cooling rate of $0.25 \mathrm{~K} / \mathrm{hr}$ is specified. The velocity is initialized to the geostrophic wind throughout the domain. The virtual potential temperature is initialized at $265 \mathrm{~K}$ from the surface up to $100 \mathrm{~m}$. Above that, a capping inversion with a strength of $0.01 \mathrm{~K} / \mathrm{m}$ is specified. Random fluctuations with an amplitude of $0.1 \mathrm{~K}$ are added to the initial temperature field below $50 \mathrm{~m}$. The reference temperature is set to $\theta_{0}=263.5 \mathrm{~K}$. The surface aerodynamic roughness height is $z_{0}=0.1 \mathrm{~m}$. The flow is computed for $9 \mathrm{~h}$ of simulation time with averages taken over the last hour and in the planes of homogeneity.

Two SGS models are tried: the standard Smagorinsky model with the model constant set to three different values $\left(C_{s}=0.1,0.135\right.$, and 0.17$)$ and the LASI dynamic Smagorinsky model. The LASI quantities, for which partial-differential equations are solved, are initially set to $\mathscr{I}_{L M}=2.56 \times$ $10^{-6} \mathrm{~m}^{4} / \mathrm{s}^{4}$ and $\mathscr{I}_{M M}=1.0 \times 10^{-4} \mathrm{~m}^{4} / \mathrm{s}^{4}$ uniformly throughout the field such that the Smagorinsky constant is initially $C_{s}=0.16$. The turbulent Prandtl number is fixed uniformly throughout the field at a value of 1 .

Figure 2 shows the mean vertical profiles of wind speed and direction and potential temperature from these stable ABL simulations. The right-hand column shows the results from our simulations, and the left-hand column shows those from the GABLS intercomparison. The different colored lines in the GABLS intercomparison plots each represent a simulation performed by a different group that participated in the intercomparison. Those interested in more detail should see Ref. [29]. In all cases, a wind speed profile is predicted that has a low-level jet. In other words, the speed reaches a value about $20 \%$ greater than geostrophic speed near the top of the boundary layer. There is also a considerable wind direction change, about $40^{\circ}$, over the height of the boundary layer. The most notable point is that the simulations using the LASI dynamic Smagorinsky model predict a considerably deeper boundary layer than do those using the standard Smagorinsky model or those of the GABLS intercomparison. As resolution is increased, though, the boundary layer depth is reduced somewhat when using the LASI dynamic Smagorinsky model. The majority of the GABLS intercomparison profiles have a low-level jet peak just below $200 \mathrm{~m}$, similar to our simulations with the standard Smagorinsky model. This led us to explore the behavior of the predicted model constant $C_{s}$ from the LASI dynamic Smagorinsky model, which is discussed in depth below. In short, we found the constant $C_{s}$ to be fairly noisy, having sharp spikes with values reaching roughly 4.6. Therefore, we applied a bounding on the model constant such that its value is clipped if greater than 0.14 and if less than 0.07 . We chose these values because they are roughly the minimum and maximum of the time-averaged values of $C_{s}$ from our simulations using the LASI dynamic Smagorinsky model. The velocity profile from the simulation using the bounded LASI dynamic Smagorinksy model is much more similar to the GABLS participants results and to the standard Smagorinsky model results. Similar conclusions can be drawn by observing the mean potential temperature profiles-the LASI dynamic Smagorinsky model causes the initial temperature profile to be affected to a greater height than with the other cases, and it predicts the near surface temperature to be higher than in the other cases, but bounding the value of $C_{S}$ produces results more in line with the standard Smagorinsky and GABLS participant results. Changing the value of $C_{s}$ with the standard Smagorinsky model has surprisingly little effect on the mean profiles.

Figure 3 shows resolved velocity variance profiles from the simulations of the present study and those of the GABLS model intercomparison. In general, the horizontal variances predicted in our 

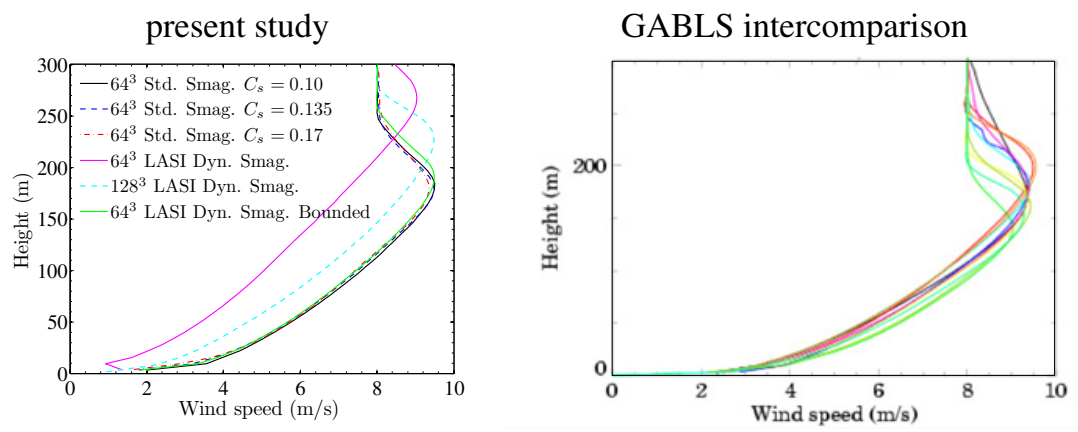

(a)
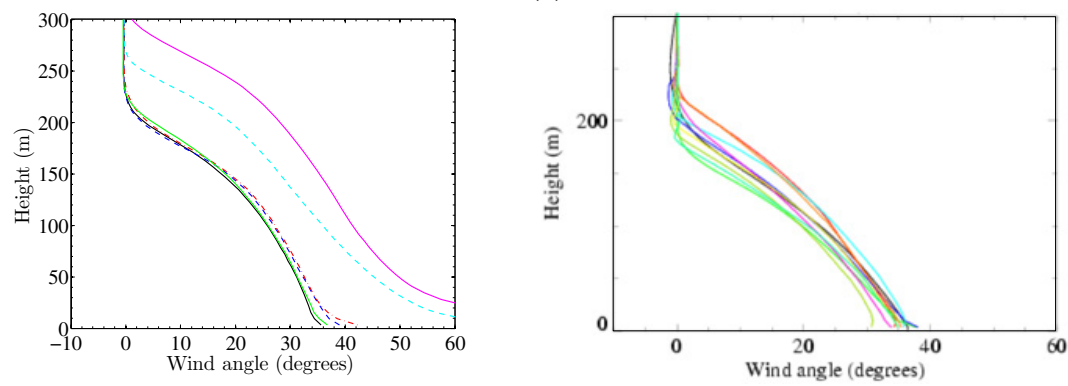

(b)
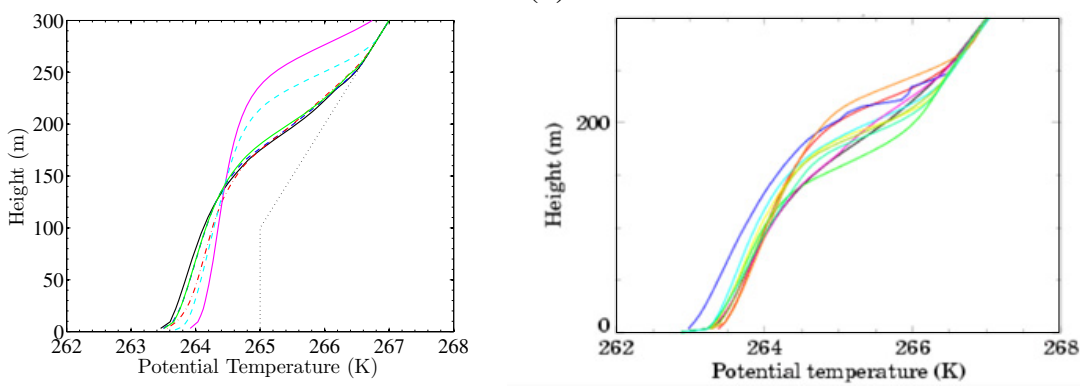

(c)

Figure 2. Mean vertical profiles of (a) wind speed, (b) wind direction, and (c) potential temperature from simulation of the present study (left column) and the GABLS model intercomparison (right column). All GABLS intercomparison figures are from Reference [29].

simulations are similar to those of larger magnitude from the GABLS intercomparison. The predictions using the LASI dynamic Smagorinksy model, though, greatly overpredict the horizontal variances. Bounding the value of $C_{s}$, however, produces a horizontal variance profile much more like the GABLS results but with a slightly larger peak value and very similar to the profile produced when using the standard Smagorinsky model with $C_{s}=0.1$.

The resolved vertical variances predicted by the standard Smagorinksy model are quite similar to those of the GABLS intercomparison. As the constant $C_{s}$ is increased in our standard Smagorinsky cases, the peak variance value decreases and the location of the peak increases in height. Again, surprisingly, the LASI dynamic Smagorinsky model predicts variances that are far greater than in the standard Smagorinsky or GABLS intercomparison cases. As resolution is increased, though, the LASI dynamic Smagorinsky model predicts smaller values of peak variances. Bounding the value of $C_{s}$ with the LASI dynamic Smagorinksy model, though, greatly reduces the magnitude of the vertical variance 

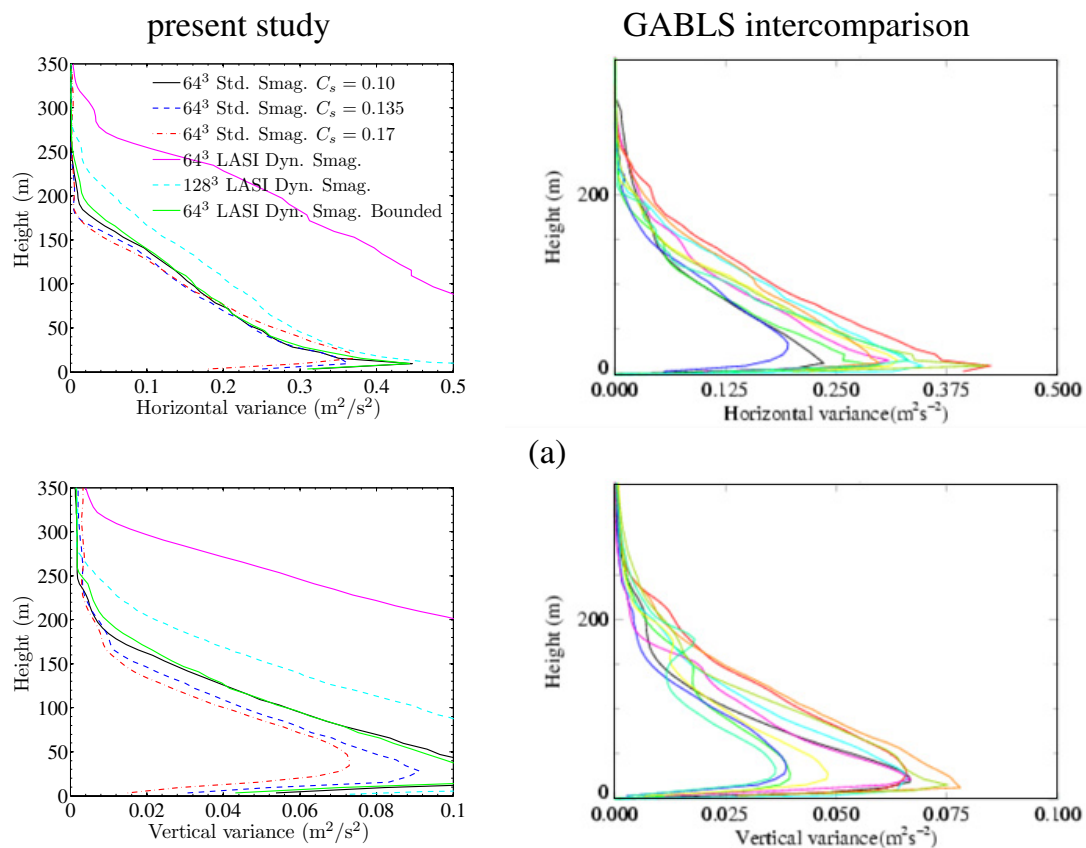

(a)

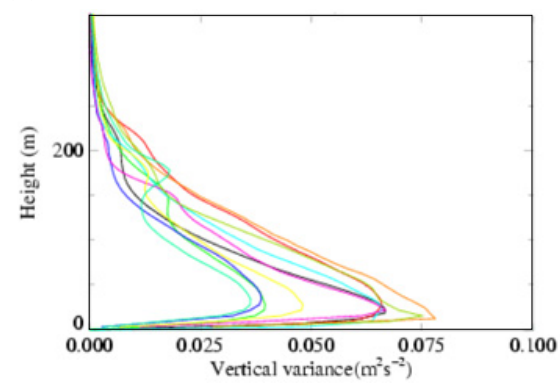

(b)

Figure 3. Vertical profiles of (a) horizontal and (b) vertical resolved velocity variance from simulation of the present study (left column) and the GABLS model intercomparison (right column). All GABLS intercomparison figures are from Reference [29].

profile and produces a profile very similar to that from the case using the standard Smagorinksy model with $C_{s}=0.1$.

Figure 4 shows contours of instantaneous velocity fluctuations in the $x$-direction in a horizontal plane at $3.125 \mathrm{~m}$ (passing through the cell centers of the lower surface-adjacent cells in the $64^{3}$ cases). Three main observations can be made. First, the peak magnitude of the fluctuations predicted with the LASI dynamic Smagorinsky model are much greater than those predicted with the standard Smagorinsky model, which is consistent with the much greater peak velocity variances predicted with that model as seen in Figure 3. Second, bounding the model constant $C_{s}$ predicted by the LASI dynamic Smagorinksy model reduces the peak velocity fluctuations to levels between those of the results from using the standard Smagorinksy model with $C_{s}=0.1$ and 0.135 . Last, with the standard Smagorinsky cases, as the value of $C_{s}$ is increased, the contours of velocity fluctuation become smoother and show less of the smaller-scale content, although their peak values do not noticeably change.

To further assess the LASI dynamic Smagorinsky model, we plot the time-averaged vertical profile of $C_{s}$, which we denote as $\left\langle C_{s}\right\rangle$, in Figure 5a. Over the entire profile, the values of $\left\langle C_{s}\right\rangle$ are on the low side of the range of values of $C_{s}$ typically used with the standard Smagorinsky model. Adding bounding of the value of $C_{s}$ causes even lower values of $\left\langle C_{s}\right\rangle$; within the lower part of the boundary layer $\left\langle C_{s}\right\rangle$ is roughly 0.115 . When resolution is doubled, the values of $\left\langle C_{s}\right\rangle$ at a given height generally drop slightly. The cause of the kink in the profile below about $25 \mathrm{~m}$ is unclear. It is possible that the value of $\left\langle C_{s}\right\rangle$ reduces near the surface because shear dominates and dynamic models reduce $C_{s}$ in the presence of shear. Below that local minimum, though, $C_{s}$ increases back to 0.135 and 0.12 for the $64^{3}$ cell nonbounded and bounded cases, respectively. This increase could be caused by some sort of surface-induced effect, interaction with the surface stress model, or inappropriate specification of the 

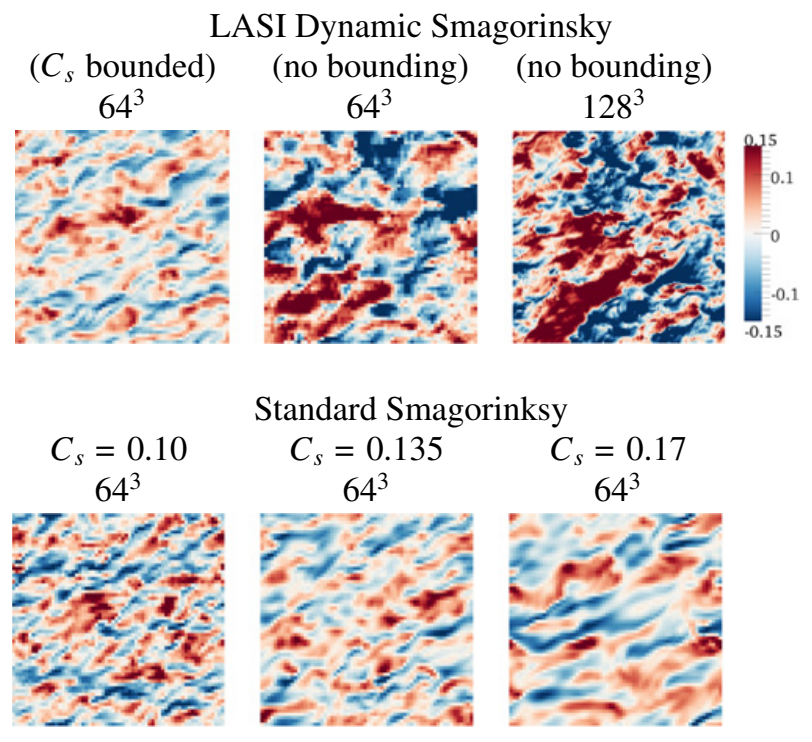

\section{Standard Smagorinksy}

$$
C_{s}=0.135 \quad C_{s}=0.17
$$

$64^{3}$

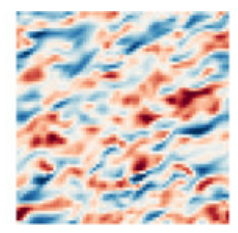

$64^{3}$

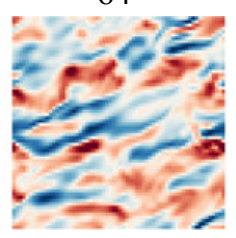

Figure 4. Contours of instantaneous velocity fluctuations $(\mathrm{m} / \mathrm{s})$ in the $x$-direction in a horizontal plane $3.125 \mathrm{~m}$ above the lower surface.

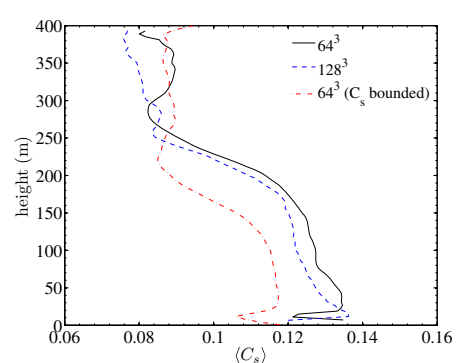

(a)

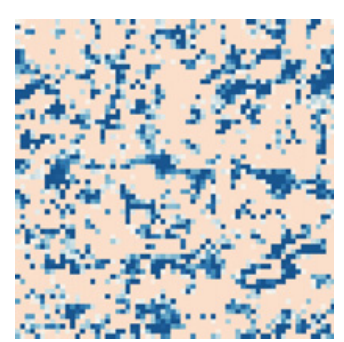

(b)

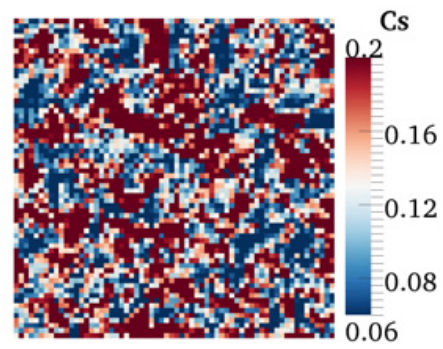

(c)

Figure 5. (a) Profiles of mean $C_{s}$ and contours of instantaneous $C_{s}$ in a horizontal plane $3.125 \mathrm{~m}$ above the lower surface from the $64^{3}$ simulation using the LASI dynamic Smagorinsky model with (b) and without (c) $C_{s}$ bounding.

vertical gradient of velocity in the centers of the lower-surface-adjacent cells. Figures $5 \mathrm{~b}$ and $\mathrm{c}$ show the instantaneous values of $C_{s}$ in cells in a horizontal plane at $3.125 \mathrm{~m}$ above the surface from the $64^{3}$ cell cases with and without bounding, respectively. The instantaneous values of $C_{s}$ are noisy. For the unbounded case, the minimum and a maximum values of $C_{s}$ are 0.0035 and 4.6, respectively, in this horizontal plane (the color scale covers a narrower range to show the majority of the values). It may be plausible that this noise acts somewhat like backscatter. In the unbounded case, the backscatter effect is excessive causing the SGS stresses to contain fluctuations that cause the strong velocity variance seen in Figure 3.

\subsection{Terrain}

We focused on a single terrain case in order to test our implementation of the local (does not use planar averaged velocities in the Monin-Obukhov scaling laws) surface stress model in terrain-local 


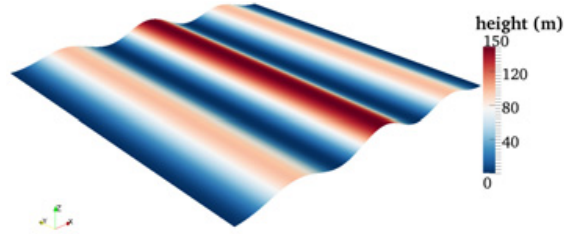

(a)

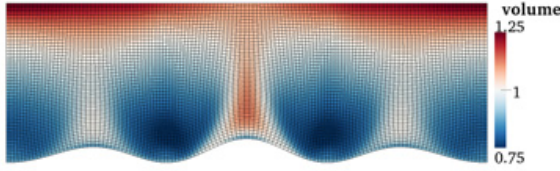

(b)

Figure 6. (a) The surface of the simply hilly terrain described by Eq. (26) and colored by contours of height. (b) A view of an $x-z$ plane of the simple hilly terrain mesh colored by contours of cell volume normalized by the volume if the mesh were uniformly spaced with no terrain, highlighting region of mesh expansion (red) and compression (blue).

coordinates. In this case, a $3 \mathrm{~km} \times 3 \mathrm{~km} \times 1 \mathrm{~km}$ domain in the $x$-, $y$-, and $z$-directions is used. The domain is periodic in the lateral directions; wall conditions, as described above, are applied at the lower surface; and a rigid, stress-free lid is used on the top boundary. The mesh size is $125 \times 125 \times 50$, which is relatively coarse but suitable for this somewhat qualitative study. The atmospheric stability is set to neutral with the potential temperature initialized to $300 \mathrm{~K}$ up to $800 \mathrm{~m}$ above which a strong capping inversion is placed in which the temperature rises to $308 \mathrm{~K}$ in the next $100 \mathrm{~m}$. Above that, a weaker inversion with a strength of $0.01 \mathrm{~K} / \mathrm{m}$ is specified. The reference temperature is set to $\theta_{0}=300 \mathrm{~K}$. The geostrophic wind is set to $U_{g}=(15.0,0.0,0.0) \mathrm{m} / \mathrm{s}$ and the simulation takes place at $45^{\circ}$ north latitude. The surface aerodynamic roughness height is $z_{0}=0.16 \mathrm{~m}$. The simulation is run for $4.5 \mathrm{~h}$ with the averages taken over the last $2.5 \mathrm{~h}$. Only the standard Smagorinsky model with $C_{s}=0.135$ is used.

The terrain consists of three two-dimensional hills with ridge lines perpendicular to the geostrophic wind, although the wind near the hill tops does not cross them perpendicularly. The terrain, which is shown in Figure 6a is described by

$$
z=\frac{A}{2} \exp \left[-\left(\frac{x-x_{\text {mid }}}{\epsilon}\right)^{2}\right]\left[1+\cos \left(2 \pi n \frac{x-x_{\text {mid }}}{x_{\text {max }}-x_{\text {min }}}\right)\right],
$$

where $A=150 \mathrm{~m}, x_{\text {min }}=0 \mathrm{~m}, x_{\text {max }}=3000 \mathrm{~m}, x_{\text {mid }}=1500 \mathrm{~m}$, and $n=3$. The mesh is initially created with uniform resolution using OpenFOAM's blockMesh tool, and then OpenFOAM's moveDynamicMesh tool is used to deform the mesh to the terrain using the "displacementSBRStress" solver with the diffusivity of the solver set to quadratic using the inverse distance from the lower surface.

Our analysis is simple and is meant to qualitatively verify the proper behavior of the flow and our method. Figure 7 shows contours of mean velocity and resolved-scale turbulent kinetic energy in a vertical plane perpendicular to the terrain ridge lines. Figure 7a shows the $x$-component of the mean flow. The top of the boundary layer is clearly visible and reversed flow is seen on the lee side of the hills. The reversed flow is caused by flow separation and recirculation. The $y$-component of the mean flow shown in Figure $7 \mathrm{~b}$ shows that the flow turns with elevation due to Coriolis accelerations. There is no $y$-velocity above the boundary layer, but below it there is significant $y$-velocity. Interestingly, in the separation/recirculation region, the value of the mean $y$-velocity is smaller than elsewhere at the same elevation. Figure 7c shows the vertical component of velocity and recirculation of flow back up the lee side of the hills is evident. Figures $7 \mathrm{~b}$ and $\mathrm{c}$ show strange velocity behavior in the layer of cells adjacent to the lower surface. There appears to be a large change in these components of the flow between these first layer cells and the cells farther from the surface. Possibly this is an effect of using no non-orthogonal corrector steps and no non-orthogonal corrections to the discretization. As expected, Figure 7d shows that there is increased turbulent kinetic energy behind the hills.

To qualitatively verify the behavior of the surface stress model, we plot (color contours) the $x$-component of instantaneous velocity in the cells adjacent to the surface in Figure 8 . We also plot 


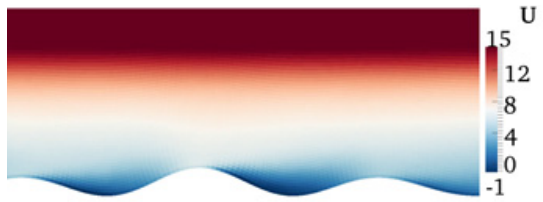

(a)

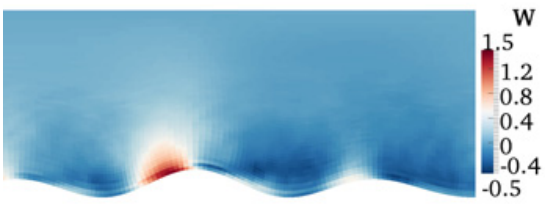

(c)

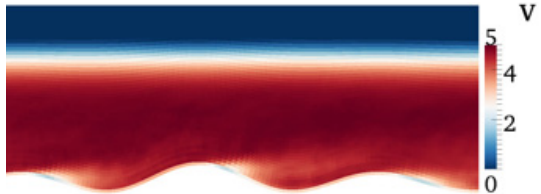

(b)

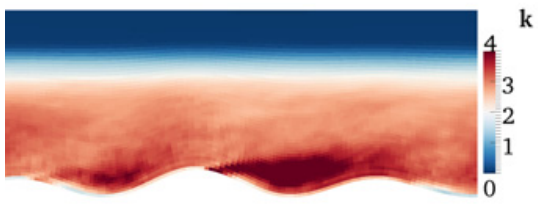

(d)

Figure 7. Contours of the (a) $x$-, (b) $y$-, and (c) $z$-components of mean velocity (m/s) and (c) resolved-scale turbulent kinetic energy $\left(\mathrm{m}^{s} / \mathrm{s}^{s}\right)$ in a vertical plane perpendicular to the terrain ridge lines.

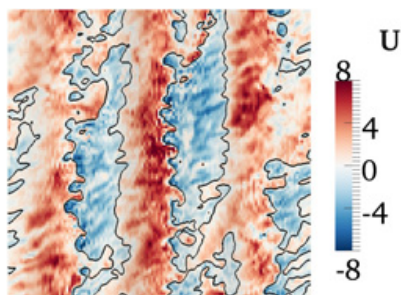

Figure 8. Contours (colored) of the $x$-component of instantaneous velocity $(\mathrm{m} / \mathrm{s})$ in cells adjacent to the hilly surface and the contour (black lines) of where the $x$-directed component of instantaneous surface shear stress is zero.

(black line) the contour of where the $x$-directed component of the surface shear stress is zero. Surface shear stress equal to zero can be an indicator of separated flow. Figure 8 shows that the locations where the flow separates (where its velocity goes to zero and then reverses) are well correlated with the contour of zero shear stress.

\section{Conclusion and future work}

This document presents our initial work at adding the capability to NREL's OpenFOAM-based SOWFA flow solver to simulate stably stratified atmospheric boundary layer flow and flow over complex terrain. Before this work, SOWFA's handling of the SGS terms in the momentum and potential temperature equations was not the same as in all other OpenFOAM solvers, so it was not compatible with the SGS models that come standard with OpenFOAM. We have migrated SOWFA back to the OpenFOAMstandard SGS treatment and can use models like the LASI dynamic Smagorinsky model. Also, before this work, the surface stress and temperature flux models included with SOWFA were formulated in such a way that they were only compatible with flat terrain. Now, these models have the ability to work in terrain-local coordinates, and the Monin-Obukhov scaling laws used by the models can be applied locally.

We applied our new method to the stably stratified ABL GABLS model intercomparison test case using both the standard and LASI dynamic Smagorinsky models. Surprisingly, the standard Smagorinsky model, which is often viewed as too dissipative for stably stratified flows in which 
turbulence levels are low and/or intermittent, produced mean vertical profiles of wind speed, direction, and potential temperature and variance profiles very similar to the simulation results of the GABLS intercomparison. The LASI dynamic Smagorinsky results were much different. That SGS model caused a much deeper boundary layer (roughly $300 \mathrm{~m}$ versus less than $200 \mathrm{~m}$ ) and far greater peak velocity variances. Contours of instantaneous velocity fluctuations show much stronger fluctuations in the LASI dynamic Smagorinsky cases as compared to the standard Smagorinsky cases. We found that the model produces spikes of $C_{s}$ with values as high as 4.6, so we applied bounding to the value of $C_{s}$. If greater than 0.14 or less than $0.07, C_{s}$ is clipped. The bounded LASI dynamic Smagorinksy results are much more in line with the GABLS participant results and the standard Smagorinksy results for $C_{s}=0.1$. A possible next step is to implement one of the LASD dynamic Smagorinsky model variants, which have been shown in the literature to produce better results than the LASI variant.

In simulating the GABLS case in which surface temperature cooling rate is specified, rather than surface temperature flux itself, we found it difficult to obtain a meaningful friction velocity and surface temperature flux to create the desired cooling rate. Specifically, solving for the friction velocity and temperature flux requires finding the roots of a polynomial, and normally the largest positive root is chosen for friction velocity. Depending on the flow situation, we found there to be many durations in the simulation in which the only solution was that friction velocity is zero, which is unrealistic. We need to remedy this problem in future work.

Next, we applied our new method to flow over an idealized hilly terrain under neutral atmospheric stratification. This was only a qualitative study, but it did confirm that the localized, terrain-local surface stress formulation goes to zero and switches sign when moving into a region of flow separation. More quantitative testing of our terrain methods is necessary, and we plan to simulate cases for which experimental data are available such as the Askervein [30] and Bolund hills.

We also plan to reexamine the use of local Monin-Obukhov scaling in the surface stress and temperature flux models. Monin-Obukhov scaling was derived from average velocity, temperature, and moisture profiles, so using the scaling locally does not necessarily make sense. The scaling laws were also derived for flow over relatively flat terrain. We should not expect this scaling (i.e. the log-law) to hold over complex terrain. Another option is to use detached-eddy simulation in which a Reynoldsaveraged Navier-Stokes model handles the region near the surface that the surface stress and temperature flux models currently handle. Yet one more option is to apply some sort of local terrain curvature correction to the standard scaling laws.

We would like to acknowledge Sukanta Basu and Yao Wang for their help and advice in simulating the stably stratified ABL. We would like to thank David Lapoint-Thériault for his useful ideas in implementing the surface stress model. We also would like to thank Marshall Buhl, Andy Platt, Billy Hoffman, and Rodd Hamann for their help with the computer resources used in this study. This work was supported by the U.S. Department of Energy under Contract No. DE-AC36-08GO28308 with the National Renewable Energy Laboratory.

\section{References}

[1] M.J. Churchfield, S. Lee, P.J. Moriarty, L.A. Martínez, S. Leonardi, G. Vijayakumar, J.G. Brasseur, A Large-Eddy Simulation of Wind-Plant Aerodynamics, in 50th AIAA Aerospace Sciences Meeting including the New Horizons Forum and Aerospace Exposition, Nashville, TN, Jan. 9-12, 2012 (AIAA, Washington D.C., 2012), AIAA Paper 2012-537

[2] A.R. Brown, S.H. Derbyshire, P.J. Mason, Quarterly Journal of the Royal Meteorological Society 120, 1485 (1994) 
[3] R.J. Beare, M.K. MacVean, A.A.M. Holtslag, J. Cuxart, I. Esau, J.C. Golaz, M.A. Jimenez, M. Khairoutdinov, B. Kosovic, D. Lewellen et al., Boundary-Layer Meteorology 118, 247 (2006)

[4] R. Stoll, F. Porté-Agel, Water Resources Research 42, W01409 (2006)

[5] R. Stoll, F. Porté-Agel, Boundary-Layer Meteorology 126, 1 (2008)

[6] F. Wan, F. Porté-Agel, R. Stoll, Atmospheric Environment 41, 2719 (2007)

[7] C. Meneveau, T. Lund, W. Cabot, Journal of Fluid Mechanics 319, 353 (1996)

[8] N.D. Kelley, Tech. Rep. TP-5000-52353, National Renewable Energy Laboratory (2011)

[9] J. Smagorinksy, Monthly Weather Review 91, 99 (1963)

[10] C.H. Moeng, Journal of the Atmospheric Sciences 41, 2052 (1984)

[11] P.J. Mason, S.H. Derbyshire, Boundary-Layer Meteorology 53, 117 (1990)

[12] P.J. Mason, D.J. Thompson, Journal of Fluid Mechanics 242, 51 (1992)

[13] P.P. Sullivan, J.C. McWilliams, C.H. Moeng, Boundary-Layer Meteorology 71, 247 (1994)

[14] A. Andren, Quarterly Journal of the Royal Meteorological Society 121, 961 (1995)

[15] B. Kosović, Journal of Fluid Mechanics 336, 151 (1997)

[16] B. Kosović, J.A. Curry, Journal of the Atmospheric Sciences 57, 1052 (2000)

[17] M. Germano, U. Piomelli, P. Moin, W. Cabot, Physics of Fluids A 3, 1760 (1991)

[18] F. Porté-Agel, C. Meneveau, M.B. Parlange, Journal of Fluid Mechanics 415, 261 (2000)

[19] E. Bou-Zeid, C. Meneveau, M. Parlange, Physics of Fluids 17, 025105 (2005)

[20] S. Basu, F. Porté-Agel, Journal of the Atmospheric Sciences 63, 2074 (2006)

[21] R.J. Beare, M.K. MacVean, Boundary-Layer Meteorology 112, 257 (2004)

[22] J. Kleissl, V. Kumar, C. Meneveau, M.B. Parlange, Water Resources Research 442, W06D10 (2006)

[23] P.P. Sullivan, E.G. Patton, K.W. Ayotte, Turbulent flow over and around sinusoidal bumps, hills, gaps and craters derived from large eddy simulations, in 19th Conference on Boundary Layer and Turbulence, Keystone, CO, Aug. 2-6, 2010 (2010), paper 1B.5

[24] G. Kirkil, J. Mirocha, E. Bou-Zeid, F.K. Chow, B. Kosović, Monthly Weather Review 140, 266 (2012)

[25] D. Lapoint-Thériault, Ph.D. thesis, École De Technologie Supérieure (2012)

[26] P.J. Oliveira, R.I. Issa, Numerical Heat Transfer, Part B 40, 473 (2001)

[27] U. Schumann, Journal of Computational Physics 18, 376 (1975)

[28] S. Basu, A.A.M. Holtslag, B.J.H.V.D. Weil, A.F. Moene, G.J. Steeneveld, Acta Geophysica 56, 88 (2008)

[29] GABLS LES intercomparison, http://gabls.metoffice.com/index.html (2006)

[30] P.A. Taylor, H.W. Teunissen, Tech. Rep. Report Number MSRB-84-6, Research Canada (1985), available online at http://www.yorku.ca/pat/research/Askervein/index.html 